\title{
Competencias sociales y linguíisticas en las fuentes primarias
}

El taller "Historiador/a por un día"

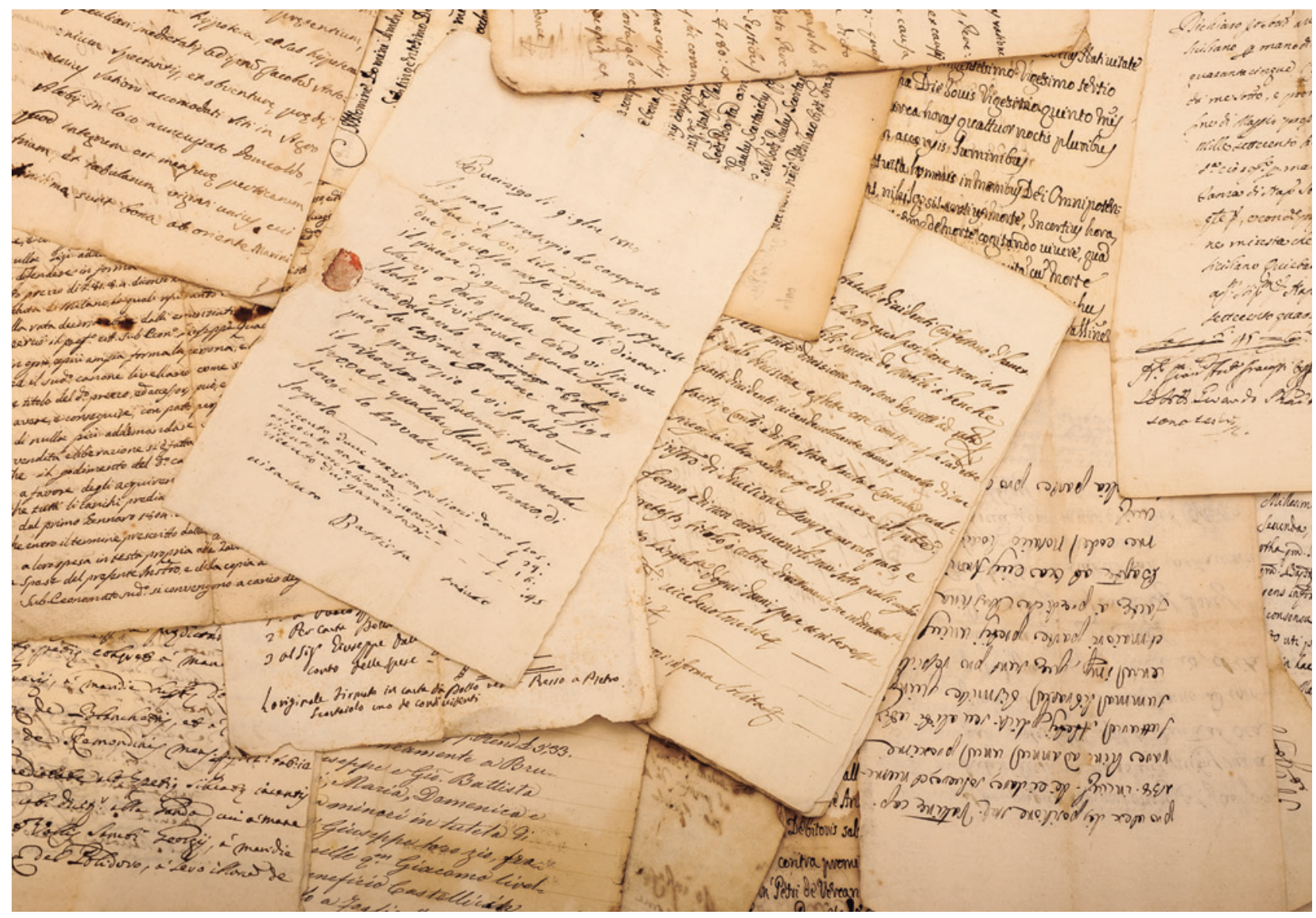

A través de un taller didáctico de paleografía, se analiza una fuente primaria para calibrar la adquisición y la utilización de las competencias lingüísticas, sociales y cívicas en el alumnado de Educación Primaria o

José Ignacio Ortega Cervigón

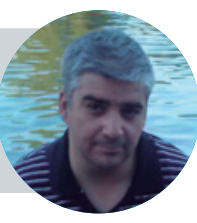

Universidad Complutense de Madrid Facultad de Educación-CFP joseiort@ucm.es

Secundaria. La actividad se estructura en apartados que, por medio de unas preguntas sencillas, imitan el método científico desarrollado por el historiador y estimulan la participación activa de los alumnos. 


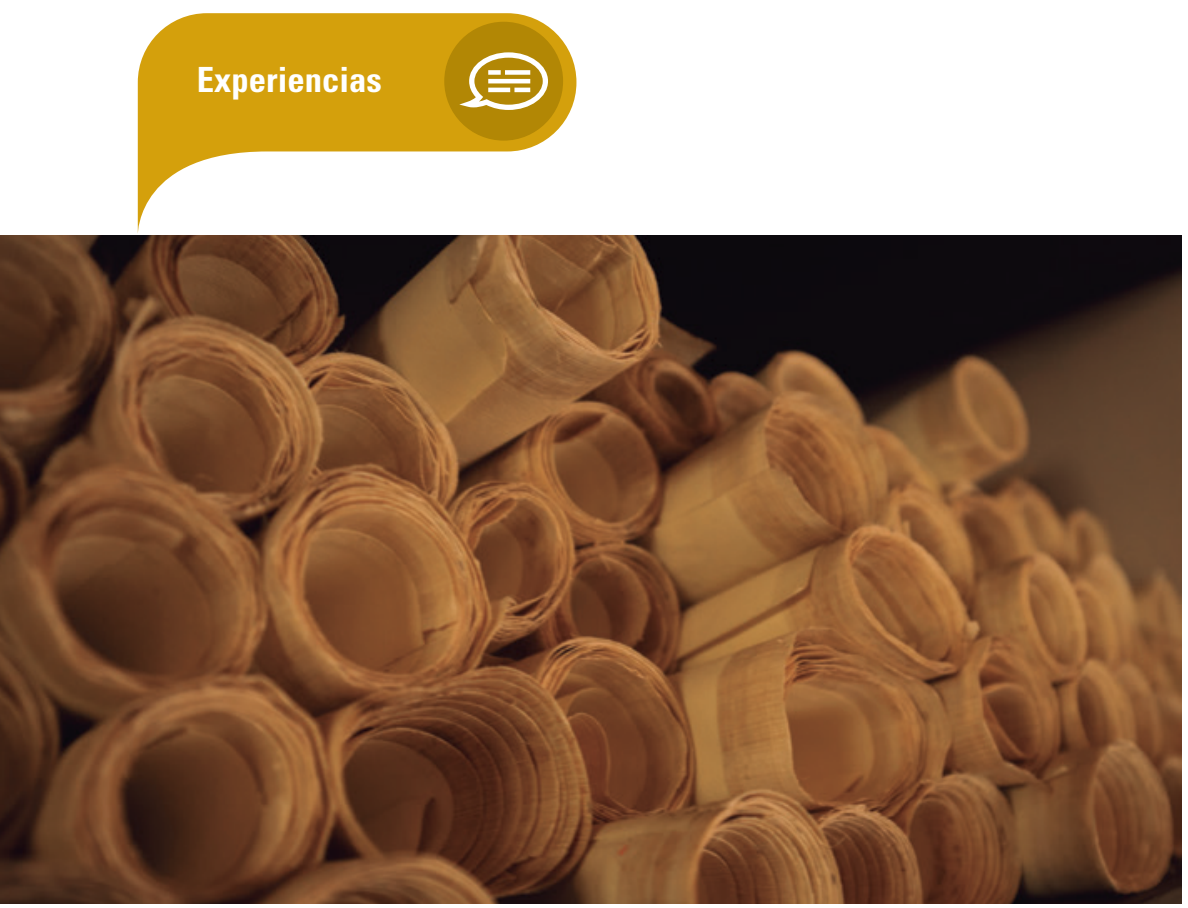

El recurso a las fuentes primarias en la explicación didáctica de la historia ha quedado reflejado en diversos estudios como una vía altamente recomendable para obtener un rendimiento óptimo en el aprendizaje significativo de los alumnos de las etapas de Educación Primaria y Secundaria. El análisis y el comentario de textos documentales permiten adquirir varias de las competencias básicas recogidas en el currículo actual, en especial la comunicación lingüística, la autonomía e iniciativa personal y la competencia social y ciudadana.

La explotación didáctica de una fuente histórica, a través de una serie de preguntas pautadas, logra acercar el método científico a los alumnos para generar conocimiento sobre aspectos políticos, institucionales, sociales, económicos y culturales. Por tanto, la utilización de fuentes primarias puede proporcionar unas herramientas de trabajo que contribuyan a la creación del pensamiento histórico en los alumnos y alumnas, que profundiza en los contenidos conceptuales y asimila procesos y habilidades para obtener conocimientos en un contexto de aprendizaje significativo.

\section{Fuentes primarias y competencias}

El tratamiento de la información de documentos históricos y fuentes primarias permite desarrollar competencias básicas y claves del proceso de enseñanzaaprendizaje. Dentro del componente lingüístico, el taller planteado potencia aspectos relacionados con el léxico, la semántica, la ortografía, la comprensión lectora de textos y la comunicación constructiva. Además, podemos analizar a través de las fuentes el grado de dominio de la comunicación lingüística del alumnado como fórmula de expresión, canalización y argumentación de dicho conocimiento.

En los componentes de las competencias sociales y cívicas la fuente contribuye a la comprensión de los códigos de conducta en sociedades del pasado, así como el aprendizaje de conceptos como sociedad, igualdad, no discriminación entre mujeres y hombres, democracia o ciudadanía. Las fuentes documentales son un excelente punto de partida para formular hipótesis sobre la construcción del conocimiento histórico y las distintas variables sociales y la comprensión del concepto de multicausalidad.

\section{El uso de las fuentes primarias en Historia}

Para el proceso de aprendizaje del tiempo histórico es muy importante contar con recursos que plasmen de forma efectiva los cambios, por ejemplo, en las viviendas, los transportes o la escritura. Los docentes de ciencias sociales debemos ofrecer visiones diacrónicas amplias y contextualizar los hechos caracterizando los procesos sociales por su estructura económica, ideológica, cultural y política. El contexto histórico ayuda a la comprensión de los hechos y las diversas transformaciones que se van generando.

El trabajo con fuentes primarias facilita al alumno conocer las variables de los procesos sociales, el concepto abstracto de multicausalidad y el relativismo del propio conocimiento histórico. Otro aspecto didáctico destacable es favorecer la capacidad empática con sociedades alejadas en el tiempo e intentar obtener respuestas sobre el funcionamiento interno de una sociedad, cuya comparación con estructuras anteriores y posteriores ofrece la detección de cambios y permanencias.

La explicación de los procesos sociales ha de tener un sentido crítico. La utilización de fuentes en el aula permite observar las argumentaciones, relaciona procesos a través de causas y consecuencias y com- 
prende las transformaciones anejas a los hechos expuestos. Los documentos muestran protagonistas, formas de vida cotidiana, conflictos sociales, instituciones, creencias o variables educativas: un fondo de recursos en los que descubrir pervivencias o transformaciones del pasado. Esta comparación con el presente posibilita la detección de cambios y permanencias y dota a los alumnos de las herramientas para construir una narración histórica al desarrollar procedimientos de formulación y comprobación de hipótesis, y cotejarlas con las conclusiones ofrecidas por las corrientes historiográficas.

Otras funciones que cumplen los documentos son la toma de conciencia del pasado como transmisor de conocimientos, así como la preocupación por conservar el patrimonio de las colectividades humanas. Por último, el alumnado puede alcanzar un sentido crítico al dudar de la veracidad de lo que está recogido en el escrito y poder plantear la construcción de un conocimiento histórico alternativo. Los alumnos, por tanto, pueden iniciarse en los procedimientos esenciales del método científico y afianzar los conceptos básicos del aprendizaje del tiempo histórico, como el cambio, la continuidad, la duración, la sucesión, la simultaneidad o la multicausalidad.

\section{Taller "Historiador/a por un día": objetivos}

A través del taller "Historiador/a por un día" se analiza una fuente primaria que permite calibrar la adquisición, la utilización y el afianzamiento de la competencia lingüística y las competencias sociales y cívicas en el alumnado de Ciencias Sociales. La introducción de fuentes primarias con fines didácticos se puede realizar con alumnos y alumnas de 8-10 años, que han adquirido las competencias de lectoescritura. No obstante, en la franja de 10 a 12 años ya pueden trabajar con fuentes de mayor complejidad y han de realizar interrelaciones entre el documento y los conocimientos adquiridos sobre el contexto histórico que se trabaje para obtener sencillas deducciones de in-

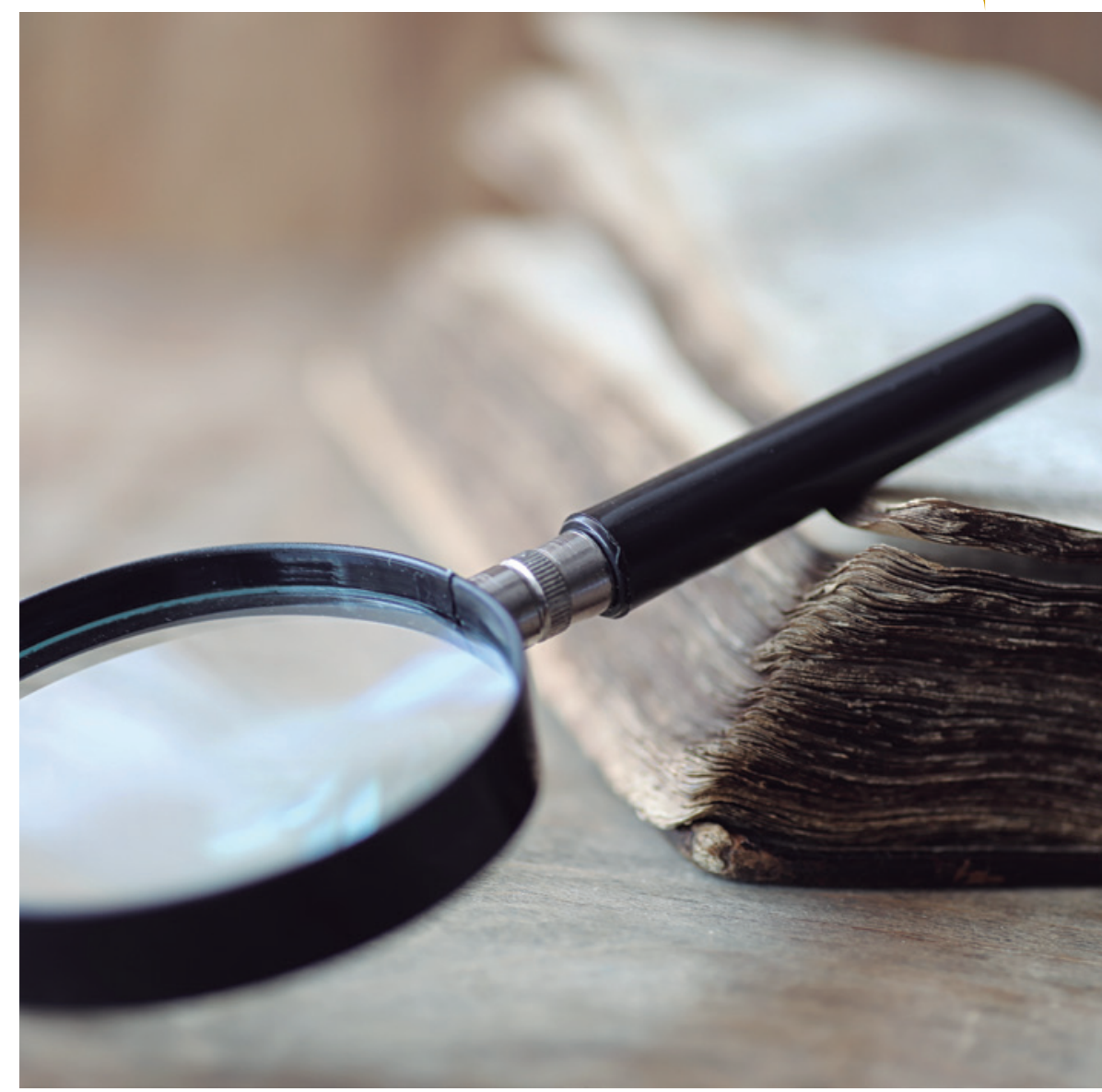

formación. Las fuentes escritas, así como las materiales, explican aspectos de las sociedades del pasado en diversos aspectos: institucional, económico, social, tecnológico, cultural, cotidiano, etc.

Entre los objetivos didácticos del taller "Historiador/a por un día" se encuentran los siguientes:

入 Acercamiento a las ciencias auxiliares en la enseñanza de la historia, como la paleografía.

入 Utilización de fuentes primarias para la generación de conocimiento histórico en el aula a través de preguntas indagatorias.

入 Adquisición de pensamiento social e histórico a través de la utilización del método científico del historiador y la comparación con la sociedad actual.

入 Alcance de altas cuotas de desarrollo en las argumentaciones de la causalidad de los procesos históricos.

入 Comprensión del tiempo histórico mediante la comparación de grupos sociales del pasado con la sociedad actual y la observación y el análisis de los cambios y las permanencias existentes. 


\section{Metodología didáctica del taller}

La metodología de la propuesta didáctica es el desarrollo - en un centro escolar de Primaria o Secundaria- de un taller de paleografía básico que analiza una fuente primaria. En su realización se incluye una aplicación de cuál es el método científico en las Ciencias Sociales: ¿cómo trabajan los historiadores y las historiadoras? ¿Cuáles son las principales fuentes de información que utilizan?

La explicación previa al taller se inicia con el concepto de fuente histórica, que constituye la materia prima de la historia. Las fuentes comprenden todos los documentos, testimonios u objetos

\section{Las fuentes escritas son el apoyo básico para la construcción de conocimiento histórico. Los investigadores trabajan las fuentes históricas y las interrogan para obtener de ellas la mayor información posible}

que nos trasmiten una información significativa referente a los hechos que han tenido lugar, especialmente en el pasado. Dentro de ellas, y considerando el valor que también tienen las demás, las fuentes escritas son el apoyo básico para la construcción de conocimiento histórico. Los investigadores trabajan las fuentes históricas -las interrogan y contrastan con otros testimonios - para obtener de ellas la mayor información posible y deben realizar una adecuada selección de las mismas.

Existen dos tipos de fuentes históricas: primarias, que han sido elaboradas de forma simultánea a los hechos que estamos investigando y no han sido modificadas, y secundarias o historiográficas, que se nutren de otras fuentes primarias o secundarias. Seguidamente, se expone al alumnado las diferentes categorías de fuentes por su origen: escritas, ma- teriales, gráficas y orales, mencionando varios ejemplos de cada una de ellas. La finalidad es mostrar la variedad de documentos y evitar algunas confusiones: una fuente documental no es evidentemente solo la que está escrita y en un soporte como el papel o el pergamino.

A continuación, se explica el trabajo metodológico de los historiadores, recordando someramente que la Historia es una disciplina científica. Como toda ciencia, el método histórico requiere de un proceso de selección y recopilación de fuentes, de análisis y comparación de datos y de conclusiones que corroboran o refutan las hipótesis iniciales. Para la confección del conocimiento histórico, las fuentes utilizadas deben ser analizadas, valoradas e interpretadas, siguiendo una metodología coherente. Asimismo, las fuentes han de ser sometidas a una crítica objetiva para conocer los elementos que las componen y comprobar su veracidad. El objetivo de este proceso es, por tanto, comprender cómo se lleva a cabo la construcción de la Historia.

Para una comprensión sencilla y remarcando la distancia que los separa, se puede equiparar el trabajo de los historiadores al de los arqueólogos y al de los periodistas, cuyas profesiones se basan en la búsqueda de pruebas, evidencias e información a través de preguntas. Es muy útil recurrir a las seis "W" que toda noticia periodística recoge para el alcance completo de sus objetivos: what?, when?, where?, who?, why? y how? Los historiadores, de la misma forma, pueden realizar una serie de preguntas a la fuente documental para extraer información histórica.

Además de utilizar el método científico que desarrollan los historiadores, el taller es una pequeña iniciación al conocimiento de la paleografía, ciencia que estudia las distintas escrituras antiguas y su evolución a lo largo del tiempo. Aunque es complejo explicar cuestiones técnicas del método paleográfico, el alumnado es capaz de apreciar las distintas grafías que adoptan las letras a partir de los ejemplos seleccionados. Se aportan unas tablas que recogen la evolución del alfabeto castellano a través de una serie de cua- 
dros que recogen las letras minúsculas y mayúsculas entre los siglos XII y XVII. Por último, se muestra una copia digital de la fuente primaria, un documento histórico extraído del Archivo de Villa de Madrid.

\section{La fuente documental como herramienta de trabajo}

A través de estos interrogantes acercamos a los alumnos a la temática en la que se puede encuadrar el documento que informa sobre hechos y sucesos. Así realizaremos las preguntas siguientes:

त What? Identificamos los hechos y las situaciones históricas que ocurren. La tipología de la fuente y la apariencia formal puede ayudar a señalar la temática central del documento. La primera cuestión estriba en definir qué es el documento. Un padrón de pecheros es una fuente de naturaleza fiscal que expone un listado de personas que deben pagar impuestos y la cuantía que aporta cada una. Es sencillo comparar la fuente con un censo de población o un padrón municipal, cuyos datos quedan registrados por los ayuntamientos.

$\boldsymbol{\lambda}$ When? El documento está fechado en 1497, una época gozne entre la Edad Media y la Edad Moderna. También es interesante hilar el documento con el tiempo cronológico y preguntar por acontecimientos relevantes de esa época.

त Where? En numerosas ocasiones, el lugar suele ir acompañado de la fecha y es en sí mismo un dato fundamental para caracterizarlo. El padrón de pecheros es de la aldea de Getafe, perteneciente a la jurisdicción de la Villa de Madrid. Después de Madrid, era el principal núcleo de población de su tierra y a finales del siglo XV contaba con unos dos mil habitantes.

$\lambda$ Who? Los protagonistas que aparecen son los vecinos pecheros, es decir, los que pagaban impuestos. Por tanto, nos encontramos ante una fuente que se ubica en el contexto de una sociedad estamental. Los alumnos han de describir la existencia de estamentos pri-

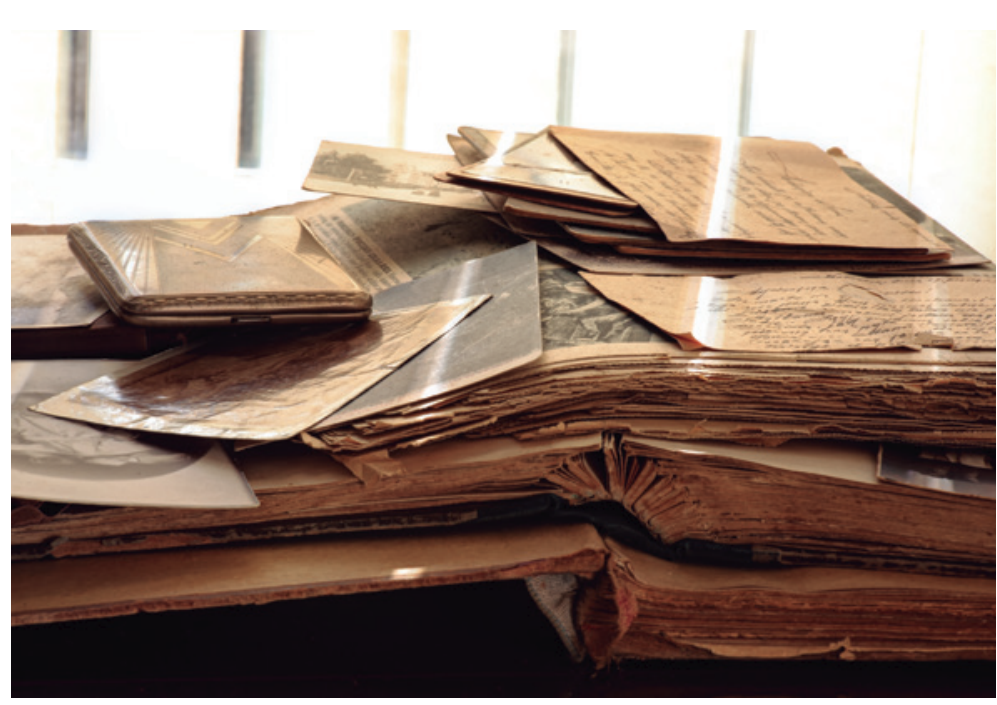

vilegiados — grupos sociales exentos del pago de impuestos, el clero y la nobleza-y no privilegiados, que eran la mayor parte de la sociedad e incluía a comerciantes, artesanos, campesinos y ganaderos. Se puede comentar que una persona equivalía a una unidad fiscal, es decir, a una familia nuclear de 4 o 5 miembros.

त El análisis meticuloso de los nombres y apellidos de las personas censadas ofrece mucha información interesante, tanto desde el punto de vista de la competencia lingüística como de la competencia social y cultural. Casi la totalidad de los nombres propios remiten a un contexto religioso que tamiza las mentalidades de la época. Se pueden extraer los principales nombres por un análisis cuantitativo: Juan, Pedro, Fernando, Marcos y Miguel eran los más habituales.

$\boldsymbol{\lambda}$ Respecto al escaso porcentaje de mujeres, los alumnos han de visualizar su situación de discriminación política, social y familiar. Aunque sus actividades primordiales eran las labores domésticas, la crianza de los hijos y algunos trabajos agrícolas, hubo mujeres que trabajaron como artesanas y algunas se hacían cargo de la administración de los bienes familiares. En el documento aparecen pocas mujeres cuyo reflejo es el nombre de pila o la viuda de fulano.

त Why? La aldea de Getafe se regía por un concejo similar al de la Villa de Madrid y este era la institución destinataria de recaudar los pagos concejiles. La recaudación de estos ingresos se desti- 
naba a satisfacer determinadas rentas de la corona, como las alcabalas y las tercias, y al gasto generado por la burocracia concejil, los pleitos del lugar y las reformas urbanas. El concejo es el antecedente histórico de los ayuntamientos municipales.

7 How? Se deben analizar todos los aspectos históricos destacados que muestra y describir las formas de vida que se pueden observar, así como precisar aspectos de actividades que pueden ser de diversa naturaleza: políticas, sociales, económicas, culturales, religiosas, etc. Aunque la información que ofrece el padrón a priori es limitada, se pueden extraer parámetros sobre las condiciones de vida de la época en algunos aspectos, como determinadas actividades económicas artesanales, reflejadas en los apellidos de muchas personas (sastre, herrero, zapatero); la situación de desigualdad de las mujeres; las diferencias económicas de las cuantías pagadas, que indican la existencia de algunas familias con mayor poder adquisitivo y cuyos miembros eran quienes obtenían cargos concejiles y componían la oligarquía local; etc.

El uso de las fuentes primarias, en primer lugar, conduce a la creación de pensamiento histórico en los alumnos. En segundo término, el pensamiento histórico crítico se profundiza en contenidos conceptuales y a través de la asimila-

\section{DARA SABER MÂS}

Gómez Carrasco, C. J., Ortuño Molina, J., y Miralles Martínez, P. (2018). Enseñar ciencias sociales con métodos activos de aprendizaje. Reflexiones y propuestas a través de la indagación. Barcelona: Octaedro.

Sallés Tenas, N. (2014). La introducción de las fuentes primarias en los materiales de enseñanza-aprendizaje de la historia. Íber. Didáctica de las Ciencias Sociales, Geografía e Historia, (78), 44-52.

Sandoya Hernández, M. A. (2016). Enseñar Ciencias Sociales. 35 actividades para desarrollar capacidades. Barcelona: Universitat Oberta de Catalunya. ción de procedimientos. La inclusión de documentos primarios en el proceso de aprendizaje de la historia ayuda a la adquisición de las competencias básicas, cuya interiorización supone una utilización habitual de contenidos procedimentales a través de la variedad de recursos y materiales que permiten su evaluación posterior. Es cierto, no obstante, que el análisis de fuentes requiere una "lectura disciplinar" previa para poder "escribir históricamente", labor docente más compleja al incluir vínculos intertextuales y explicaciones relacionales.

El trabajo con fuentes implica la utilización didáctica de numerosos conceptos del tiempo histórico: el ámbito de aplicación (social), el origen (lineal), la inserción en la temporalidad humana (la relación entre el pasado y el presente), las cualidades del cambio (una coyuntura económica y una estructura social), la concreción de los cambios (el criterio de evolución y desarrollo) y los elementos de dominio y gestión del tiempo (instrumentos de medida y explicación causal e intencional de los hechos). Se puede obtener una visión de cambio y transformación si los alum. nos relacionan los datos de la fuente, que reflejan las características de una sociedad preindustrial, con la realidad social en la que ellos se desenvuelven.

En definitiva, el tratamiento de la información de documentos históricos y fuentes primarias permite desarrollar competencias básicas y claves del proceso de enseñanza-aprendizaje de las Ciencias Sociales con un alto grado de motivación en el alumnado •

HEMOS HABLADO DE

\section{Didáctica de la Historia; competencia lingüística y social; fuentes primarias; taller de paleografía.}

Este artículo fue solicitado por PADRES Y MAESTROS en marzo de 2020, revisado y aceptado en junio de 2020. 